\title{
Intensity output and effectiveness of light curing units in dental offices
}

\author{
Baharan-Ranjbar Omidi ${ }^{1}$, Armin Gosili ${ }^{2}$, Mona Jaber-Ansari ${ }^{3}$, Ailin Mahdkhah ${ }^{4}$ \\ ${ }^{1}$ Assistant Professor, Department of Operative Dentistry, Faculty of dentistry, Qazvin University of Medical science, Qazvin, Iran \\ ${ }^{2}$ Assistant Professor, Department of Orthodontics, Faculty of dentistry, Golestan University of Medical science, Gorgan, Iran \\ ${ }^{3}$ Dentist, Private Practice, Tehran, Iran \\ ${ }^{4}$ Post-graduate Student of Operative Dentistry, Faculty of Dentistry, Qazvin University of Medical Science, Qazvin, Iran
}

Correspondence:

Department of Operative Dentistry

Faculty of Dentistry, Qazvin University of Medical Science

Qazvin, Iran

mahdkhahailin@yahoo.com

Received: 20/02/2018

Accepted: 11/04/2018

Omidi BR, Gosili A, Jaber-Ansari M, Mahdkhah A. Intensity output and effectiveness of light curing units in dental offices. J Clin Exp Dent. 2018;10(6):e555-60.

http://www.medicinaoral.com/odo/volumenes/v10i6/jcedv10i6p555.pdf

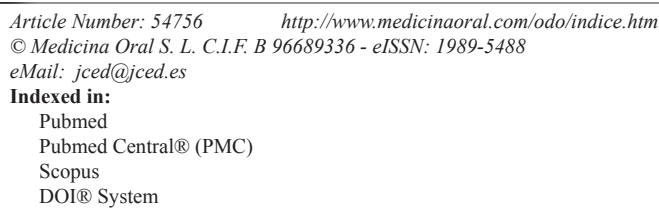

\begin{abstract}
Background: The aims of the study were measuring the light intensity of light curing units used in Qazvin's dental offices, determining the relationship between the clinical age of these units and their light intensity, and identifying the reasons for repairing them.

Material and Methods: In this cross-sectional study, the output intensity of 95 light curing devices was evaluated using a radiometer. The average output intensity was divided up into four categories (less than 200, 200-299, 300500 , and more than $500 \mathrm{~mW} / \mathrm{cm}^{2}$ ). In addition, a questionnaire was designed to obtain information mainly about the type, clinical age, and frequency of maintenance of the units and the reasons for fixing them. Data were analyzed using Kolmogorov-Smirnov, chi-squared, and t-tests $(p<0.05)$ on SPSS 24.

Results: A total of 95 light curing units were examined, with 61 (64.2\%) of them being of the LED type and 34 (35.8\%) of the QTH type. While average light intensity in LED units was significantly higher than in QTH devices, the two device types were not significantly different regarding desirable light intensity (i.e., $\geq 300 \mathrm{mw} / \mathrm{cm}^{2}$ ). A negative correlation was observed between clinical age and light intensity. In addition, bulb replacement in QTH devices was over three times as much as in LED units. Also, repairing QTHs was more than twice as much frequent as fixing LEDs. The most common reason for repair was the breakage of the tip of the device.

Conclusions: The light intensity of LED units is significantly higher than that of QTH devices, and the frequency of repairing in QTHs was significantly more than in LEDs. Furthermore, light intensity decreases with aging, and dentists should regularly monitor the conditions of light units.
\end{abstract}

Key words: Light curing unit, radiometer, light intensity, dental equipment, dental offices. 


\section{Introduction}

Over three decades have passed since the beginning of the extensive use of composite resins in dentistry, and the demand for using esthetic restorative materials is still on the increase (1). Resins must begin polymerization in order to perform operation. During this process, monomer units bond with each other to build long and heavy polymers. Due to the increased use of optical composites, the importance of polymerization has become more prominent. The strength of these restorations depends on the degree of polymerization of composite resins. Incomplete polymerization produces adverse biological effects, increasing water absorption, composite solubility, and reducing hardness. Various factors contribute to the polymerization of the composites, and they include the wavelength and intensity of the output of light curing units, duration of radiation, dimensions and location of the dental cavity, direction and distance of the tip of the device (related to the composite), the composition of the composite, the wavelength and bandwidth of the curing light, the intensity of the curing light, the irradiation time, and color and thickness of the composite $(2,3)$. In composite resins, camphorquinone is the light-sensitive component, which responds to irradiation by creating free radicals and initiates the polymerization process (4). An appropriate intensity of light with the maximum absorption wavelength range of camphorquinone is the main factor in the polymerization of these resins. If the light output intensity decreases, it will adversely influence the clinical and cosmetic performance. The light intensity of curing devices is defined by the International Organization for Standardization as the ISO 4049 standard, which recommends an intensity of $300 \mathrm{~mW} / \mathrm{cm}^{2}$ with a wavelength bandwidth of 400-515 $\mathrm{nm}$ on the tip of the light curing device. At this standard wavelength, the minimum depth of cure is assumed to be $1.5 \mathrm{~mm}$, which is $50 \%$ of the length of the composite specimen (3). The reduction in the light intensity of the device can affect the success rate of the restorative methods via reducing the degree of convergence of composites, which leads to an increase in microleakage and recurrent caries (5).

The light source for polymerization of composite resins are available in four types: quartz-tungsten-halogen (QTH), light-emitting diode (LED), plasma arc curing (PAC), and argon laser. Halogen-based curing lamps have several limitations. One of the main disadvantages of these lamps is the high energy consumption. Only $1 \%$ of the consumed energy by these devices turns into light and almost all the remaining energy is converted into heat. The heat generated by these lamps should be eliminated, and this requires expensive thermal filters. Cooling fans are also loud and bulky. Also, the longevity of halogen lamps is short (between 40 and 100 hours) (6). In 1995, Mills and colleagues presented solid-state LED technology for the polymerization of dental materials capable of being activated with light. In LEDs, instead of hot strands as used in halogen lamps, semiconductor connections are employed to produce light. These lamps have a very long shelf life of about 1,000 hours and can withstand mechanical shocks and vibrations with very low error rates (7). LEDs are also capable of producing blue light at a wavelength of 440-480 $\mathrm{nm}$. LEDs can be cordless and are almost silent while being operated (7). In QTH and LED light curing devices, the main factors affecting the intensity of light output are: inappropriate performance of the lamp and filter, breakage and pollution of the device tip, the blurring of the bulb, the failure of electrical components, and defect in light transmitting fibers $(6,7)$. In these devices, if maintenance is not carried out routinely, after a while, there will be some problems with the lamp, fan, or power supply (8).

There are two main problems with the quality of cured resin composite in the office:

1) Composite surface hardness is not a reliable guide because even at a low light intensity, the surface can sufficiently harden while the depth of the cure is not adequate. Moreover, it is impossible for the dentist to distinguish completely-cured composite resin from the one incompletely cured using a device with a low light intensity (9).

2) The output light of the device decreases as the device is used more, but this is not detectable by the unarmed eye because sometimes a seemingly bright light is not suitable for wavelengths. Furthermore, insufficient radiation intensity is not always compensated for by prolonging exposure time (10). Therefore, a digital radiometer is needed to measure the intensity of the curing light of the units to determine when the device needs to repaired or replaced (11).

The aims of the present study were four-fold: 1) measuring the light intensity of light curing units used in the offices, 2) comparing the light intensity of LED and QTH units, 3) determining the relationship between the clinical age of these devices and their light intensity, and 4) exploring the reasons for and the frequency of repairing these units.

The findings of this study underscore the importance of timely fixing or replacing defective light curing devices, which can consequently ensure the continued quality of restorative treatments. This improvement can increase public health in the long run. It is believed that these findings can be used in the macro-planning of the health system.

\section{Material and Methods}

The following criteria were used to include private dental offices in this study: 1) the person operating the office had to be a general dentist rather than a specialist, and 2) the dentist had to routinely use composite resins for too- 
th restoration. The 2016 alphabetical listing of dentists published by the Qazvin University of Medical Sciences was used to identify dental offices located in Qazvin. All the 105 offices on the list were contacted via telephone to determine if they satisfied the criteria. Of these offices, 88 fulfilled both criteria. The dentists in charge of these latter offices were contacted by telephone to explain the rationale and methodology of the study and to obtain his or her consent. Ultimately, 70 dental offices agreed to participate. Upon this, an appointment was arranged for a visit to the office.

To measure light intensity, an analog radiometer (DigiRate, Monitex, Taiwan) with a range of 0 to $1,000 \mathrm{~mW} /$ $\mathrm{cm}^{2}$ was used. The radiometer was sent to the Laboratory of Optics at Sharif University of Technology in Tehran, Iran to confirm its performance. Once approval was received, the offices were visited to perform the measurements. At each office, a few minutes were spared to allow the radiometer to match up with the ambient temperature. Then, three measurements of light intensity were recorded for each light curing unit, and the average was reported as the final measure. At the intervals between visits, the accuracy of the radiometer was checked against a radiometer at the restorative dentistry laboratory of the Qazvin University of Medical Sciences. Light intensity of less than $300 \mathrm{~mW} / \mathrm{cm}^{2}$ was considered unacceptable (3), and a device with a light intensity of less than $200 \mathrm{~mW} / \mathrm{cm}^{2}$ was regarded unusable (12).

In addition, a questionnaire, designed by the researchers, was used to obtain information about type of light curing unit, the age of the unit, the frequency of maintenance, the reasons for repair, the date of the last repair, the number of times the bulb had been replaced and the last time this had been done, the typical duration of light irradiation at each restoration, the availability of a radiometer in the office, and the number of office hours per week.

Once the data had been collected, they were analyzed using SPSS 24 (IBM Corporation, USA, 2016). The mean and standard deviation were used to determine the mean light intensity of the light curing units. The Kolmogorov-Smirnov statistic was used to determine the normality of the distribution of the light intensity scores.
The clinical age of the device was calculated using the following equation:

Clinical age $=$ time in use (in terms of year) $\times 52$ (number of weeks in a year) $\times$ number of working days of the office per week $\times$ average number of instances of the use of the device per day $\times$ average duration of light exposure (in terms of second) at each instance of the use of the device.

Pearson product-moment (r) correlation coefficient was used to determine the correlation between the clinical age and the light intensity of the device.

Independent-samples t-test was used to compare the light intensity of LED and QTH units.

The chi-squared test was employed to find out about the correlation between device type on the one hand and the frequency of bulb replacement, the frequency of device repair, and the reason for device repair on the other hand. Statistical significance for all tests was set at an $\alpha$ level of $<0.05$ (2-tailed).

-Ethical considerations

The study was endorsed by the Research Ethics Committee of Qazvin University of Medical Sciences under the approval ID of IR.QUMS.REC.1394.642. Additionally, the information contained in this study does not mention the names of the honorable participating dentists.

\section{Results}

\section{-Device type}

A total of 95 light curing units were examined: 61 (64.2\%) were LED and 34 (35.8\%) were QTH.

-Correlation between device type and light intensity The light intensity of LED and QTH units is shown in Table 1. As can be seen, about $93 \%$ of LED devices had a desirable light intensity (i.e., $\geq 300 \mathrm{mw} / \mathrm{cm}^{2}$ ), whereas this figure is around $97 \%$ for QTH units. Results from the t-test showed that the relationship between the two variables was insignificant regarding desirable light intensity. However, average light intensity in LED units was significantly higher than in QTH devices.

-Relationship between clinical age and light intensity Pearson $r$ revealed a negative relationship between clinical age and light intensity (Table 2).

Table 1: Distribution of acceptable/nonacceptable light intensity for the curing devices under study.

\begin{tabular}{|l|c|c|c|c|}
\hline \multirow{2}{*}{$\begin{array}{l}\text { Curing } \\
\text { devices }\end{array}$} & \multicolumn{4}{|c|}{ Light intensity $\mathbf{( m W / \mathbf { c m } ^ { 2 } )}$} \\
\cline { 2 - 5 } & $<200$ nonacceptable & $\begin{array}{c}200-299 \\
\text { nonacceptable }\end{array}$ & $\begin{array}{c}300-500 \\
\text { acceptable }\end{array}$ & $\begin{array}{c}\geq 500 \\
\text { acceptable }\end{array}$ \\
\hline QTH $^{\square}$ & $0(0 \%)$ & $1(2.9 \%)$ & $15(44.1 \%)$ & $18(52.9 \%)$ \\
\hline LED $^{\downarrow}$ & $0(0 \%)$ & $4(6.6 \%)$ & $8(13.1 \%)$ & $49(80.3 \%)$ \\
\hline Total & $0(0 \%)$ & $5(5.3 \%)$ & $23(24.2 \%)$ & $67(70.5 \%)$ \\
\hline
\end{tabular}

: quartz-tungsten-halogen. ${ }^{1}$ : light emitting diode. 
Table 2: Clinical age and light intensity of light curing devices.

\begin{tabular}{|c|c|c|c|c|}
\hline \multirow{2}{*}{$\begin{array}{c}\text { Clinical age } \\
\text { (hour) }\end{array}$} & \multicolumn{3}{|c|}{ Light intensity $\left.\mathbf{( m W}^{-} \mathbf{c m}^{2}\right)$} & \multirow{2}{*}{ Total } \\
\cline { 2 - 4 } & $\mathbf{2 0 0 - 2 9 9}$ & $\mathbf{3 0 0 - 5 0 0}$ & $\mathbf{5 0 0}$ & \\
\hline $\mathbf{0 - 2 0}$ & $0(0 \%)$ & $1(1.1 \%)$ & $25(26.3 \%)$ & $26(27.4 \%)$ \\
\hline $\mathbf{2 0 - 5 0}$ & $2(2.1 \%)$ & $5(5.3 \%)$ & $19(20.0 \%)$ & $26(27.4 \%)$ \\
\hline $\mathbf{5 0 - 1 1 0}$ & $3(3.2 \%)$ & $10(10.5 \%)$ & $14(14.7 \%)$ & $27(28.4 \%)$ \\
\hline $\mathbf{1 1 0 - 1 7 0}$ & $0(0 \%)$ & $5(5.3 \%)$ & $5(5.3 \%)$ & $10(10.4 \%)$ \\
\hline $\mathbf{1 7 0 - 2 3 0}$ & $0(0 \%)$ & $1(1.1 \%)$ & $2(2.1 \%)$ & $3(3.2 \%)$ \\
\hline$>\mathbf{2 3 0}$ & $0(0 \%)$ & $1(1.1 \%)$ & $2(2.1 \%)$ & $3(3.2 \%)$ \\
\hline
\end{tabular}

-Relationship between device type and the frequency of replacing device bulbs

Table 3 shows the relationship between device type and the frequency of replacing the bulb. It is evident that bulb replacement in QTH devices was over three times as much as in LED units. The chi-squared test showed a significant relationship between the two variables.

-Relationship between device type and the frequency of device repair

The relationship between device type and the frequency of device repair is given in Table 4. According to the chi-squared test results (Table 4), the relationship between these two variables was statistically insignificant.

Table 3: Frequency of replacing the bulb in LED and QTH devices.

\begin{tabular}{|c|c|c|c|c|}
\hline \multirow{5}{*}{ 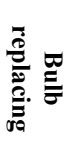 } & $\mathbf{N}$ & LED & QTH & $p$-value \\
\hline & 0 & $51(83.6 \%)$ & $14(41.2 \%)$ & \multirow{4}{*}{$\begin{array}{l}\text { 영 } \\
8\end{array}$} \\
\hline & 1 & $5(8.2 \%)$ & $14(41.2 \%)$ & \\
\hline & 2 & $4(6.5 \%)$ & $6(17.6 \%)$ & \\
\hline & 3 & $1(1.7 \%)$ & $0(0 \%)$ & \\
\hline Total & & $61(100 \%)$ & $34(100 \%)$ & \\
\hline
\end{tabular}

Table 4: Relationship between device type and the frequency of device repair.

\begin{tabular}{|c|c|c|c|c|}
\hline \multirow{5}{*}{ 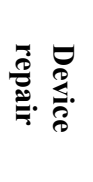 } & $\mathbf{N}$ & LED & QTH & $p$-value \\
\hline & $\mathbf{0}$ & $52(85.2 \%)$ & $24(70.5 \%)$ & \multirow{5}{*}{$\begin{array}{l}\circ \\
\stackrel{\circ}{\circ}\end{array}$} \\
\hline & 1 & $8(13.1 \%)$ & $7(20.5 \%)$ & \\
\hline & 2 & $0(0 \%)$ & $3(9.0 \%)$ & \\
\hline & 3 & $1(1.7 \%)$ & $0(0 \%)$ & \\
\hline Total & & $61(100 \%)$ & $34(100 \%)$ & \\
\hline
\end{tabular}

-Relationship between device type and the reasons for device repair

According to Table 5, the most common reason for repair was the breakage of the tip of the device. More specifically, this was the cause in $78 \%$ of all instances of repair in the case of LED devices, and $40 \%$ in the case of QTH units. The chi-squared test revealed a significant relationship between the two variables.

The devices studied are based on the model presented in Table 6. The most common model used in dentistry offices in Qazvin is the Woodpecker model, which has a good radiation intensity in all cases. It was also found that the Gnatus light cure device had the lowest percentage of light-intensity.

\section{Discussion}

This study found that most of the devices were LED-type. Despite the popularity of halogen devices in the past, there are some problems with QTHs. As noted above, these include the short life span of halogen lamps and the fact that the reflector and filter can undergo a reduction in efficiency over time. Overheating is one of the other disadvantages of such devices (6). However, these issues are less frequently seen in LEDs, which has resulted in a rapid growth of the use of these units. Another upside of LEDs is that their light output wavelength peak of $456 \mathrm{~nm}$ matches the absorption peak of camphorquinone (13). However, initiators other than camphorquinone have slightly different absorption spectra. Thus, it is better to use halogen devices with these ma-

Table 5: Relationship between device type and the reasons for device repair.

\begin{tabular}{|c|c|c|c|c|c|c|c|}
\hline \multirow[t]{2}{*}{ Device } & \multicolumn{7}{|c|}{ Reason for device repair } \\
\hline & $\begin{array}{l}\text { Device tip } \\
\text { breakage }\end{array}$ & $\begin{array}{l}\text { Blown } \\
\text { fuse }\end{array}$ & $\begin{array}{c}\text { Fan } \\
\text { failure }\end{array}$ & $\begin{array}{c}\text { Cord } \\
\text { change }\end{array}$ & $\begin{array}{c}\text { Power switch } \\
\text { failure }\end{array}$ & Total & $p$-value \\
\hline LED & $7(11.4 \%)$ & $0(0 \%)$ & $0(0 \%)$ & $0(0 \%)$ & $2(3.3 \%)$ & $9(14.7 \%)$ & \multirow[t]{2}{*}{0.029} \\
\hline QTH & $4(11.7 \%)$ & $2(5.8 \%)$ & $3(8.8 \%)$ & $1(2.9 \%)$ & $0(0 \%)$ & $10(29.4 \%)$ & \\
\hline
\end{tabular}


Table 6: Models of light curing units used in Qazvin's dental offices (2017).

\begin{tabular}{|c|c|c|c|c|c|c|c|c|c|c|c|c|c|c|c|c|c|c|}
\hline $\begin{array}{r}\text { Light } \\
\text { intensity }\end{array}$ & 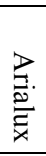 & 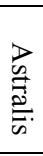 & 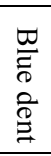 & 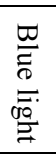 & 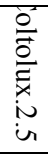 & 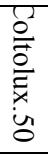 & 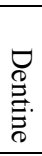 & 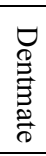 & 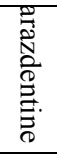 & $\begin{array}{l}\text { T. } \\
\overrightarrow{0} \\
\hat{N} \\
0 \\
0 \\
0\end{array}$ & $\begin{array}{l}\Xi_{\tilde{\aleph}} \\
\text { 苛 }\end{array}$ & $\stackrel{5}{\stackrel{\vec{P}}{x}}$ & 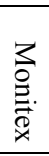 & 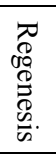 & $\sum_{2}^{\infty}$ & $\frac{\Omega}{\lambda}$ & 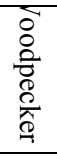 & $\overrightarrow{0}$ \\
\hline $200-299$ & 0 & 0 & 0 & 0 & 1 & 0 & 0 & 0 & 0 & 0 & 4 & 0 & 0 & 0 & 0 & 0 & 0 & 5 \\
\hline $300-500$ & 1 & 0 & 0 & 0 & 4 & 6 & 3 & 3 & 1 & 0 & 2 & 0 & 0 & 0 & 0 & 3 & 0 & 23 \\
\hline$>\mathbf{5 0 0}$ & 0 & 1 & 1 & 2 & 5 & 4 & 3 & 13 & 1 & 2 & 0 & 1 & 2 & 1 & 1 & 1 & 29 & 67 \\
\hline Total & 1 & 1 & 1 & 2 & 10 & 10 & 6 & 16 & 2 & 2 & 6 & 1 & 2 & 1 & 1 & 4 & 29 & 95 \\
\hline
\end{tabular}

terials, but they may not fully cured with LEDs because the light output wavelength range of halogen is wider than that of LEDs. On the other hand, spectral purity in LED devices allows better polymerization of composites with camphorquinone. In addition, LEDs do not emit too much heat (14).

The first aim of the present study was measuring the light intensity of light curing units in use in the dental offices of the city of Qazvin. It was found that none of the devices had a light intensity of less than $200 \mathrm{~mW} /$ $\mathrm{cm}^{2}$ and that $6.6 \%$ of LEDs, $2.9 \%$ of QTHs, and $5.3 \%$ of all total devices had a light intensity of less than 300 $\mathrm{mW} / \mathrm{cm}^{2}$, the desirable level. Unlike this study, Miyazaki et al. (10), Barghi et al. (1994), (6) and Martin (15) reported high percentages: $41.9 \%, 29.7 \%$ and $27 \%$, respectively.

In 2009, Javaheri and Ashreghi (16) concluded that the light intensity of $27.4 \%$ of the devices in their study was less than desirable. Since that study was also carried out in Qazvin, the much lower percentage reported in the present study can be attributed to the increased awareness among the dentists of the necessity of continued maintenance of light curing units over recent years. On the other hand, our findings are similar to those of Barghi et al., (11) and Savadi Oskoee (8), who reported an undesirable percentage of $10.4 \%$ and $10 \%$, respectively. Overall, the wide variety in the results reported in the literature can be ascribed to the variety in the devices in terms of type, maintenance, and clinical age. Indeed, the clinical age of $94 \%$ of devices in the present study did not exceed 170 hours. Another possible cause is the cleanliness of the tip of the devices as research has shown that the gradual buildup of the debris of composite resins on the curing tip can significantly decrease light intensity $(12,17)$. This was not measured in the present study.

Another observation was that the light intensity of LEDs is significantly higher than that of QTHs, which may be due to the difference in the output light spectrum and clinical age of devices. This finding is consistent with a study by Hegde et al. (18).

It was also observed that the clinical age of the devices is negatively correlated with the intensity of light. A similar finding was reported by Barghi et al. (6), Martin (15), Poulos and Styner (19) and Friedman (20). However, Javaheri and Ashreghi (16) concluded that there is no significant correlation between clinical age and light intensity. Two possible reasons for the difference in the results can be the use of different models of light curing devices in different studies and different levels of device consciousness on the part of participating dentists.

Furthermore, a significant relationship was found between the frequency of bulb replacement and the type of device, with greater frequency in the case of QTHs ( $58.8 \%$ as opposed to $16.4 \%$ for LEDs). This is attributable to the longer life span of an LED lamp, which is over 1,000 hours as opposed to 100 hours for a QTH lamp (7).

Also, the relationship between the frequency of lamp replacement and light intensity did not teach significance. However, it should be noted that only a small number of devices in our study had their lamp replaced. It is worth noting at this point that more frequent bulb replacement is an indicator of the aging of the device, which in turn signals the aging of the other parts of the device as the potential factors contributing to light intensity. The study also found that the main reason for repair was the breakage of the tip of device. This clearly shows the vulnerability of the tip to breakage.

Finally, the most common model used in the dental offices of Qazvin was Woodpecker (Woodpecker Med. Instrument, Guilin, china), which was found to have the favorable light intensity of all models. It was also found that the Gnatus model (Gnatus Medical-Dental Equipments, Brazil) has the lowest light intensity, but it should be noted that there were only a small number of Gnatus devices, which were also very old. However, the dentist's attention to the periodic measurement of the intensity of the device and doing the necessary repairs can increase the efficiency of the device.

\section{Conclusions}

This study concluded the following:

The light intensity of the light curing devices in the dental offices of Qazvin was acceptable (i.e., over $300 \mathrm{~mW} /$ $\mathrm{cm}^{2}$ ).

1. The light intensity of LEDs was significantly higher than that of QTH devices.

2. Light intensity decreases with the aging of the device. 3. The frequency of bulb replacement in QTHs was significantly higher than in LEDs. 
4. There was a significant relationship between device model and light intensity.

5. For dentists who do not carry out a regular maintenance of light curing devices, it is better to use LEDs because these devices have a higher light intensity and a longer bulb life span.

6 . Regarding the fact that reduction in light intensity can affect the success rate of restorative methods, light intensity measurement should be carried out regularly.

\section{References}

1. Dunne S, Davies B, Millar B. A survey of the effectiveness of dental light-curing units and a comparison of light testing devices. British dental journal. 1996;180:411-6.

2. El-Mowafy O, El-Badrawy W, Lewis DW, Shokati B, Kermalli J, Soliman $\mathrm{O}$, et al. Intensity of quartz-tungsten-halogen light-curing units used in private practice in Toronto. Journal of the American Dental Association (1939). 2005;136:766-73; quiz 806-7.

3. Fan PL, Schumacher RM, Azzolin K, Geary R, Eichmiller FC. Curing-light intensity and depth of cure of resin-based composites tested according to international standards. Journal of the American Dental Association (1939). 2002;133:429-34; quiz 91-3.

4. Willems G, Lambrechts P, Braem M, Celis JP, Vanherle G. A classification of dental composites according to their morphological and mechanical characteristics. Dent Mater. 1992;8:310-9.

5. Aguiar FHB, Ajudarte KF, Lovadino JR. Effect of light curing modes and filling techniques on microleakage of posterior resin composite restorations. Operative dentistry. 2002;27:557-62.

6. Barghi N, Berry T, Hatton C. Evaluating intensity output of curing lights in private dental offices. Journal of the American Dental Association (1939). 1994;125:992-6.

7. Goyal A, Jyothikiran H, Shivalinga BM. Use of Light Curing Units in Orthodontics: A Review. Annals of Dental Research. 2011;1:54-61. 8. Savadi Oskoee S, Poor Abbas R, Hafezehquran A. Evaluation of light curing units effectiveness used in clinics and private dental offices of Tabriz, 2001. Journal of Dental School Shahid Beheshti University of Medical Sciences. 2004;22:82-95.

9. Hansen EK, Asmussen E. Reliay of three dental radiometers. European Journal of Oral Sciences. 1993;101:115-9.

10. Miyazaki M, Hattori T, Ichiishi Y, Kondo M, Onose H, Moore BK. Evaluation of curing units used in private dental offices. Operative dentistry. 1998;23:50-4.

11. Barghi N, Fischer DE, Pham T. Revisiting the intensity output of curing lights in private dental offices. Compendium of continuing education in dentistry (Jamesburg, NJ : 1995). 2007;28:380-4; quiz 5-6.

12. Rueggeberg FA, Caughman WF, Curtis JW Jr, Davis HC. Factors affecting cure at depths within light-activated resin composites. American journal of dentistry. 1993;6:91-5.

13. Chen YC, Ferracane JL, Prahl SA. Quantum yield of conversion of the photoinitiator camphorquinone. Dental materials : official publication of the Academy of Dental Materials. 2007;23:655-64.

14. Althoff $\mathrm{O}$, Hartung M. Advances in light curing. American journal of dentistry. 2000;13(Spec No):77d-81d.

15. Martin FE. A survey of the efficiency of visible light curing units. Journal of dentistry. 1998;26:239-43.

16. Javaheri M, Ashreghi M. Evaluation of curing light intensity in private dental offices (2005). The Journal of Qazvin University of Medical Sciences. 2009;12:50-5.

17. Duke ES. Light-emitting diodes in composite resin photopolymerization. Compendium of continuing education in dentistry (Jamesburg, NJ : 1995). 2001;22:722-5.

18. Hegde V, Jadhav S, Aher GB. A clinical survey of the output intensity of 200 light curing units in dental offices across Maharashtra. Journal of conservative dentistry : JCD. 2009;12:105-8.

19. Poulos JG, Styner DL. Curing lights: changes in intensity output with use over time. General dentistry. 1997;45:70-3.
20. Friedman J, Hassan R. Comparison study of visible curing lights and hardness of light-cured restorative materials. The Journal of prosthetic dentistry. 1984;52:504-6.

\section{Conflict of interest}

The authors have declared that no conflict of interest exist. 